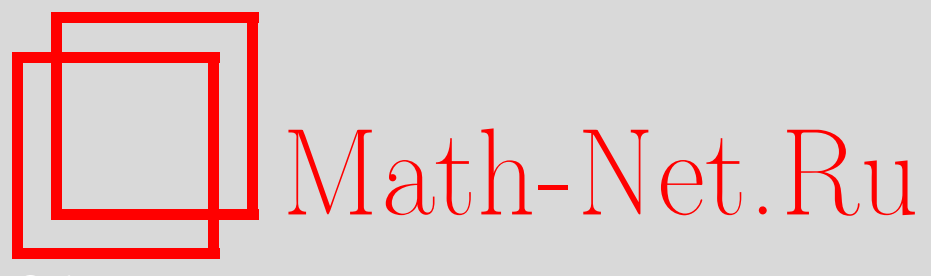

А. П. Лауринчикас, Аналог теоремы Воронина для периодических дзета-функций Гурвица, Матем. сб., 2007, том 198, номер 2, 91-102

DOI: https://doi.org/10.4213/sm1516

Использование Общероссийского математического портала Math-Net.Ru подразумевает, что вы прочитали и согласны с пользовательским соглашением http://www . mathnet.ru/rus/agreement

Параметры загрузки:

IP : 18.208 .226 .222

26 апреля 2023 г., 13:39:22

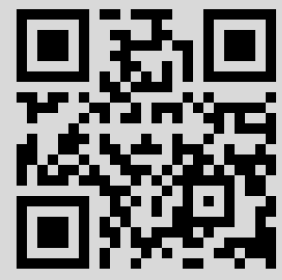




\section{А. Лауринчикас}

\section{Аналог теоремы Воронина для периодических дзета-функций Гурвица}

Получена теорема о приближении заданного набора аналитических функций сдвигами дзета-функций Гурвица с трансцендентным параметром. Этот результат является аналогом теоремы Воронина о совместной универсальности $L$-функций Дирихле.

Библиография: 28 названий.

\section{§ 1. Введение}

Пусть $\mathbb{N}, \mathbb{N}_{0}, \mathbb{R}$ и $\mathbb{C}$ - множества соответственно всех целых положительных, целых неотрицательных, действительных и комплексных чисел, а $s=\sigma+i t-$ комплексная переменная. В 1975 г. С. М. Воронин открыл замечательное свойство универсальности для дзета-функции Римана $\zeta(s)$. Он доказал в [1] (см. также [2]-[6]), что если $0<r<1 / 4$, а функция $f(s)$ непрерывна, не имеет нулей в круге $|s| \leqslant r$ и аналитична внутри этого круга, то для всякого $\varepsilon>0$ существует число $\tau=\tau(\varepsilon) \in \mathbb{R}$ такое, что

$$
\max _{|s| \leqslant r}\left|\zeta\left(s+\frac{3}{4}+i \tau\right)-f(s)\right|<\varepsilon .
$$

Теорему Воронина можно формулировать и в более общем виде. Пусть при $T>0$

$$
\nu_{T}(\ldots)=\frac{1}{T} \operatorname{meas}\{\tau \in[0, T]: \ldots\},
$$

где meas $\{A\}$ означает меру Лебега измеримого множества $A \subset \mathbb{R}$, а вместо многоточия записывается условие, которому удовлетворяет $\tau$. Тогда (см., например, [7]) если $K$ - компактное подмножество полосы $\{s \in \mathbb{C}: 1 / 2<\sigma<1\}$, обладающее связным дополнением, а функция $f(s)$ непрерывна, не имеет нулей в $K$ и аналитична внутри $K$, то для всякого $\varepsilon>0$

$$
\liminf _{T \rightarrow \infty} \nu_{T}\left(\sup _{s \in K}|\zeta(s+i \tau)-f(s)|<\varepsilon\right)>0 .
$$

Теорема Воронина об универсальности дзета-функции Римана многими авторами была обобщена для других дзета и $L$-функций и некоторых классов рядов Дирихле (см. обзорные статьи [8]-[10]).

Первый результат о совместной универсальности - приближение набора аналитических функций сдвигами $L$-функций Дирихле - также принадлежит С. М. Воронину [3]-[6].

Работа выполнена при поддержке Государственного фонда науки и высшего образования Литвы и фонда INTAS (грант № 03-51-5070).

(C) А. ЛАУринчикас, 2007 
Теорема А. Пусть $\chi_{1}, \ldots, \chi_{n}$ - попарно неэквивалентные характеры Дирихле, $K_{1}, \ldots, K_{n}$ - компактные подмножества полосы $\{s \in \mathbb{C}: 1 / 2<\sigma<1\}$, обладаюшие связным дополнением, а функиии $f_{1}(s), \ldots, f_{n}(s)$ непрерывны, не имеют нулей соответственно в $K_{1}, \ldots, K_{n}$ и аналитичны внутри $K_{1}, \ldots, K_{n}$. Тогда для всякого $\varepsilon>0$

$$
\liminf _{T \rightarrow \infty} \nu_{T}\left(\sup _{1 \leqslant j \leqslant n} \sup _{s \in K}\left|L\left(s+i \tau, \chi_{j}\right)-f_{j}(s)\right|<\varepsilon\right)>0 .
$$

Результаты, аналогичные теореме А, независимо также были получены С. М. Гонеком [11] и Б. Багчи [12], [13]. Отметим, что совместная универсальность рядов Дирихле является более сложной проблемой, чем универсальность одного ряда. При доказательстве теоремы А существенно используется ортогональность характеров Дирихле. Поэтому результатов о совместной универсальности не так много. Совместная универсальность дзета-функций Лерха была доказана в [14], а дзета-функций Матсумото (определение см. в [15]) - в [16]. В [17] рассматривалась совместная универсальность дзета-функций некоторых параболических форм. Для сверток рядов Дирихле с характером Дирихле совместная универсальность была получена в [18] и [19]: в [18] - для одного класса рядов Дирихле с мультипликативными коэффициентами, а в [19] - для автоморфных $L$-функций. Работы [20] и [21] посвящены совместной универсальности общих рядов Дирихле.

В настоящей статье мы докажем совместную универсальность периодических дзета-функций Гурвица. Напомним определение периодической дзетафункции Гурвица. Пусть $\mathfrak{a}=\left\{a_{m}: m \in \mathbb{N}_{0}\right\}-$ периодическая с периодом $k \in \mathbb{N}$ последовательность комплексных чисел, а $\alpha, 0<\alpha \leqslant 1,-$ фиксированное число. Периодическая дзета-функция Гурвица $\zeta(s, \alpha ; \mathfrak{a})$ при $\sigma>1$ определяется рядом

$$
\zeta(s, \alpha ; \mathfrak{a})=\sum_{m=0}^{\infty} \frac{a_{m}}{(m+\alpha)^{s}} .
$$

Если $\mathfrak{a}=\{1\}$ и $k=1$, то мы получаем классическую дзета-функцию Гурвица $\zeta(s, \alpha)$, которая имеет мероморфное продолжение на всю комплексную плоскость с простым полюсом $s=1$ и вычетом 1. Легко видеть, что при $\sigma>1$

$$
\begin{aligned}
\zeta(s, \alpha ; \mathfrak{a}) & =\sum_{l=0}^{k-1} \sum_{m=0}^{\infty} \frac{a_{l}}{(m k+l+\alpha)^{s}}=\frac{1}{k^{s}} \sum_{l=0}^{k-1} a_{l} \sum_{m=0}^{\infty} \frac{1}{(m+(l+\alpha) / k)^{s}} \\
& =\frac{1}{k^{s}} \sum_{l=0}^{k-1} a_{l} \zeta\left(s, \frac{l+\alpha}{k}\right) .
\end{aligned}
$$

Равенство (1) дает аналитическое продолжение функции $\zeta(s, \alpha ; \mathfrak{a})$ на всю комплексную плоскость. Положим

$$
a=\frac{1}{k} \sum_{m=0}^{k-1} a_{m} .
$$

Если $a=0$, то функция $\zeta(s, \alpha ; \mathfrak{a})$ является целой, а если $a \neq 0$, то она в точке $s=1$ имеет простой полюс с вычетом $a$. 
Универсальность функции $\zeta(s, \alpha ; \mathfrak{a})$ рассматривалась в [22] и [23]. В [22] предполагалось, что $\min _{0 \leqslant m \leqslant k-1}\left|a_{m}\right|>0$, а в [23] удалось отказаться от этого условия.

Теорема Б (см. [23]). Пусть $\alpha$ - трансцендентное число, $K$ - компактное подмножество полосы $\{s \in \mathbb{C}: 1 / 2<\sigma<1\}$, обладающее связным дополнением, а функиия $f(s)$ непрерьвна, не имеет нулей в $K$ и аналитична внутри $K$. Тогда для всякого $\varepsilon>0$

$$
\liminf _{T \rightarrow \infty} \nu_{T}\left(\sup _{s \in K}|\zeta(s+i \tau, \alpha ; \mathfrak{a})-f(s)|<\varepsilon\right)>0 .
$$

Теперь пусть $\mathfrak{a}_{j}=\left\{a_{m j}: m \in \mathbb{N}_{0}\right\}-$ периодическая с периодом $k_{j} \in \mathbb{N}$ последовательность комплексных чисел, $\alpha, 0<\alpha \leqslant 1$, - фиксированное число и при $\sigma>1$

$$
\zeta\left(s, \alpha ; \mathfrak{a}_{j}\right)=\sum_{m=0}^{\infty} \frac{a_{m j}}{(m+\alpha)^{s}}, \quad j=1, \ldots, n .
$$

Через $k=\left[k_{1}, \ldots, k_{n}\right]$ обозначим наименьшее общее кратное чисел $k_{1}, \ldots, k_{n}$ и определим матрицу

$$
A=\left(\begin{array}{cccc}
a_{11} & a_{12} & \ldots & a_{1 n} \\
a_{21} & a_{22} & \ldots & a_{2 n} \\
\ldots & \ldots & \ldots & \ldots \\
a_{k 1} & a_{k 2} & \ldots & a_{k n}
\end{array}\right) .
$$

Положим $D=\{s \in \mathbb{C}: 1 / 2<\sigma<1\}$.

Теорема 1. Предположим, что $\operatorname{rank}(A)=n$. Пусть $\alpha-$ трансцендентное число, $K_{1}, \ldots, K_{n}$ - компактные подмножества полосъ $D$, обладающие связным дополнением, а функиии $f_{1}(s), \ldots, f_{n}(s)$ непрерывны, не имеют нулей соответственно в $K_{1}, \ldots, K_{n}$ и аналитичны внутри $K_{1}, \ldots, K_{n}$. Тогда для всякого $\varepsilon>0$

$$
\liminf _{T \rightarrow \infty} \nu_{T}\left(\sup _{1 \leqslant j \leqslant n} \sup _{s \in K_{j}}\left|\zeta\left(s+i \tau, \alpha ; \mathfrak{a}_{j}\right)-f_{j}(s)\right|<\varepsilon\right)>0 .
$$

\section{§2. Оценки роста функции $\zeta(s, \alpha ; \mathfrak{a})$}

Для дальнейшего нам будут нужны оценки функции $\zeta(s, \alpha ; \mathfrak{a})$ при $|t| \rightarrow \infty$ и ее среднего квадратического.

ЛЕмма 1. При $\sigma>1 / 2 u|t| \geqslant t_{0}>0$ имеет место оценка

$$
\zeta(\sigma+i t, \alpha ; \mathfrak{a}) \ll_{\alpha, \mathfrak{a}}|t|^{\delta}
$$

с некоторым $\delta>0$.

ДоказАтельство. Хорошо известно (см., например, [24]), что при $\sigma>1 / 2$ и $|t| \geqslant t_{0}$

$$
\zeta(\sigma+i t, \alpha)=\alpha^{-s}+O\left(|t|^{\delta_{1}}\right)
$$

с некоторым $\delta_{1}>0$. Отсюда и из равенства (1) получаем требуемую оценку леммы. 
ЛЕмма 2. При $\sigma>1 / 2$

$$
\int_{1}^{T}|\zeta(\sigma+i t, \alpha ; \mathfrak{a})|^{2} d t \ll_{\sigma, \alpha, \mathfrak{a}} T, \quad T \rightarrow \infty .
$$

ДокАЗАТЕЛЬСТво. В силу теорем 3.3 .1 и 3.3 .2 из [25] при $\sigma>1 / 2$

$$
\int_{1}^{T}|\zeta(\sigma+i t, \alpha)|^{2} d t \ll_{\sigma, \alpha} T, \quad T \rightarrow \infty .
$$

Следовательно, утверждение леммы является следствием равенства (1).

\section{§ 3. Совместная предельная теорема для функций $\zeta\left(s, \alpha ; \mathfrak{a}_{j}\right)$}

Доказательство теоремы 1 основано на совместной предельной теореме в смысле слабой сходимости вероятностных мер в пространстве аналитических функций для $\zeta\left(s, \alpha ; \mathfrak{a}_{j}\right)$.

Пусть $G$ - область на комплексной плоскости. Через $H(G)$ обозначим пространство аналитических в $G$ функций, наделенное топологией равномерной сходимости на компактах. Пусть

$$
H^{n}(G)=\underbrace{H(G) \times \cdots \times H(G)}_{n} .
$$

Через $\mathscr{B}(S)$ будем обозначать класс борелевских множеств пространства $S$. Докажем предельную теорему о слабой сходимости при $T \rightarrow \infty$ вероятностной меры

$$
P_{T}(A)=\nu_{T}\left(\left(\zeta\left(s+i \tau, \alpha ; \mathfrak{a}_{1}\right), \ldots, \zeta\left(s+i \tau, \alpha ; \mathfrak{a}_{n}\right)\right) \in A\right), \quad A \in \mathscr{B}\left(H^{n}(D)\right),
$$

с явным видом предельной меры.

Пусть $\gamma=\{s \in \mathbb{C}:|s|=1\}$ - единичная окружность на комплексной плоскости и

$$
\Omega=\prod_{m=0}^{\infty} \gamma_{m}
$$

где $\gamma_{m}=\gamma$ для всех $m \in \mathbb{N}_{0}$. В силу теоремы Тихонова $\Omega$ является компактной топологической абелевой группой. Поэтому на $(\Omega, \mathscr{B}(\Omega))$ существует единственная вероятностная мера Хаара $m_{H}$. Получаем вероятностное пространство $\left(\Omega, \mathscr{B}(\Omega), m_{H}\right)$. Через $\omega(m)$ обозначим проекцию элемента $\omega \in \Omega$ на координатное пространство $\gamma_{m}$. При $s \in D_{0}=\{s \in \mathbb{C}: \sigma>1 / 2\}$ положим

$$
\zeta\left(s, \alpha, \omega ; \mathfrak{a}_{1}, \ldots, \mathfrak{a}_{n}\right)=\left(\zeta\left(s, \alpha, \omega ; \mathfrak{a}_{1}\right), \ldots, \zeta\left(s, \alpha, \omega ; \mathfrak{a}_{n}\right)\right),
$$

где

$$
\zeta\left(s, \alpha, \omega ; \mathfrak{a}_{j}\right)=\sum_{m=0}^{\infty} \frac{a_{m j} \omega(m)}{(m+\alpha)^{s}}, \quad j=1, \ldots, n .
$$

Лемма 3. $\zeta\left(s, \alpha, \omega ; \mathfrak{a}_{1}, \ldots, \mathfrak{a}_{n}\right)$ является $H^{n}\left(D_{0}\right)$-значным случайным элементом, определенным на вероятностном пространстве $\left(\Omega, \mathscr{B}(\Omega), m_{H}\right)$. 
ДокАЗАТЕЛЬСтво. Из работы [22] следует, что $\zeta\left(s, \alpha, \omega ; \mathfrak{a}_{j}\right)$ для каждого $j=1, \ldots, n$ является $H\left(D_{0}\right)$-значным случайным элементом. Отсюда вытекает утверждение леммы.

Через $M(G)$ обозначим пространство мероморфных на $G$ функций, наделенное топологией равномерной сходимости на компактах. Пусть

$$
M^{n}(G)=\underbrace{M(G) \times \cdots \times M(G)}_{n},
$$

а $\widehat{P}_{\zeta}$ - распределение случайного элемента $\zeta\left(s, \alpha, \omega ; \mathfrak{a}_{1}, \ldots, \mathfrak{a}_{n}\right)$, т.е.

$$
\widehat{P}_{\zeta}(A)=m_{H}\left(\omega \in \Omega: \zeta\left(s, \alpha, \omega ; \mathfrak{a}_{1}, \ldots, \mathfrak{a}_{n}\right) \in A\right), \quad A \in \mathscr{B}\left(H^{n}\left(D_{0}\right)\right) .
$$

Лемма 4. Вероятностная мера

$$
\nu_{T}\left(\left(\zeta\left(s, \alpha, \omega ; \mathfrak{a}_{1}\right), \ldots, \zeta\left(s, \alpha, \omega ; \mathfrak{a}_{n}\right)\right) \in A\right), \quad A \in M^{n}\left(D_{0}\right),
$$

при $T \rightarrow \infty$ слабо сходится $к$ мере $\widehat{P}_{\zeta}$.

ДокАЗАТЕльство. Поскольку $\alpha$ - трансцендентное число, система

$$
\left\{\log (m+\alpha): m \in \mathbb{N}_{0}\right\}
$$

линейно независима над полем рациональных чисел. Кроме того, при $m \geqslant 1$

$$
\log (m+\alpha) \geqslant \log m
$$

Поэтому ввиду лемм 1 и 2 лемма 4 является следствием более общей теоремы 2 из [26], доказанной для общих рядов Дирихле.

Лемма 5. Вероятностная мера $P_{T}$ при $T \rightarrow \infty$ слабо сходится $к$ мере $P_{\zeta}$, где $P_{\zeta}-$ сужение меры $\widehat{P}_{\zeta}$ на $H^{n}(D)$.

Доказательство. Функция $h: M^{n}\left(D_{0}\right) \rightarrow M^{n}(D)$, определенная покоординатным сужением, непрерывна. Поэтому лемма вытекает из леммы 4 и свойств слабой сходимости вероятностных мер (см., например, $[27 ; \S 5])$.

\section{§ 4. О плотности одного множества}

При $s \in D$ и $a(m) \in \gamma$ рассмотрим ряд

$$
\sum_{m=0}^{\infty}\left(\frac{a_{m 1} a(m)}{(m+\alpha)^{s}}, \ldots, \frac{a_{m n} a(m)}{(m+\alpha)^{s}}\right) .
$$

При доказательстве того, что $\zeta\left(s, \alpha ; \mathfrak{a}_{j}\right), j=1, \ldots, n$, является $H\left(D_{0}\right)$-значным случайным элементом, в [22] было установлено, что ряд

$$
\sum_{m=0}^{\infty} \frac{a_{m j} \omega(m)}{(m+\alpha)^{s}}
$$


для почти всех $\omega \in \Omega$ относительно меры Хаара $m_{H}$ сходится равномерно на компактных подмножествах полуплоскости $D_{0}$. Отсюда вытекает существование последовательности $\left\{b_{m}: b_{m} \in \gamma, m \in \mathbb{N}_{0}\right\}$ такой, что ряд

$$
\sum_{m=0}^{\infty}\left(\frac{a_{m 1} b_{m}}{(m+\alpha)^{s}}, \ldots, \frac{a_{m n} b_{m}}{(m+\alpha)^{s}}\right)
$$

сходится в $H^{n}(D)$.

Лемма 6. Множество всех сходящихся рядов (2) всюду плотно в $H^{n}(D)$.

Для доказательства леммы 6 мы воспользуемся следующими утверждениями.

Лемма 7. Пусть $\left\{\left(f_{m 1}(s), \ldots, f_{m n}(s)\right): m \in \mathbb{N}_{0}\right\}$ - последовательность в $H^{n}(D)$, удовлетворяющая условиям:

1) если $\mu_{1}, \ldots, \mu_{n}-$ комплекснозначные меры на $(\mathbb{C}, \mathscr{B}(\mathbb{C}))$ с компактными носителями, содержащимися в $D$, такие, что

$$
\sum_{m=0}^{\infty}\left|\sum_{j=1}^{n} \int_{\mathbb{C}} f_{m j}(s) d \mu_{j}(s)\right|<\infty,
$$

mo

$$
\int_{\mathbb{C}} s^{r} d \mu_{j}(s)=0, \quad j=1, \ldots, n, \quad r \in \mathbb{N}_{0}
$$

2) ряд

$$
\sum_{m=0}^{\infty}\left(f_{m 1}(s), \ldots, f_{m n}(s)\right)
$$

сходится в $H^{n}(D)$

3) для любого компакта $K \subset D$

$$
\sum_{m=0}^{\infty} \sum_{j=1}^{n} \sup _{s \in K}\left|f_{m j}(s)\right|^{2}<\infty .
$$

Тогда множество всех сходящихся рядов

$$
\sum_{m=0}^{\infty}\left(a(m) f_{m 1}(s), \ldots, a(m) f_{m n}(s)\right), \quad a(m) \in \gamma,
$$

всюду плотно в $H^{n}(D)$.

Лемма 7 является частным случаем леммы 6 работы [17].

ЛЕмма 8. Пусть $\mu$ - комплекснозначная мера на $(\mathbb{C}, \mathscr{B}(\mathbb{C}))$ с компактным носителем, содержашимся в полуплоскости $\left\{s \in \mathbb{C}: \sigma>\sigma_{0}\right\}$,

$$
f(z)=\int_{\mathbb{C}} e^{s r} d \mu(s), \quad z \in \mathbb{C},
$$

u $f(z) \not \equiv 0$. Тогда

$$
\limsup _{r \rightarrow \infty} \frac{\log |f(r)|}{r}>\sigma_{0}
$$

Доказательство см. в [7; лемма 6.4.10]. 
Теперь пусть $A$ - подмножество множества натуральных чисел, обладающее положительной плостностью, т.е.

$$
\lim _{x \rightarrow \infty} \frac{1}{x} \#\{m \in A: m \leqslant x\}>0 .
$$

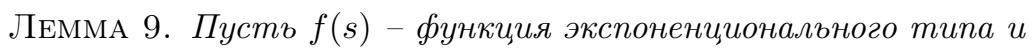

$$
\limsup _{r \rightarrow \infty} \frac{\log |f(r)|}{r}>-1 .
$$

Тогда

$$
\sum_{m \in A}|f(\log m)|=\infty .
$$

Доказательство дано в [14; лемма 5].

ДокАЗАТЕЛЬСтво ЛЕммы 6. Ясно, что достаточно доказать, что множество всех сходящихся рядов

$$
\sum_{m=0}^{\infty}\left(\frac{a_{m 1} b_{m} a(m)}{(m+\alpha)^{s}}, \ldots, \frac{a_{m n} b_{m} a(m)}{(m+\alpha)^{s}}\right)
$$

с $a(m) \in \gamma$ всюду плотно в $H^{n}(D)$. Для этой цели проверим условия леммы 7 для последовательности

$$
\left\{\left(\frac{a_{m 1} b_{m}}{(m+\alpha)^{s}}, \ldots, \frac{a_{m n} b_{m}}{(m+\alpha)^{s}}\right): m \in \mathbb{N}_{0}\right\} .
$$

Условие 2) леммы 7 имеет место в силу выбора $\left\{b_{m}: m \in \mathbb{N}_{0}\right\}$. Пусть $K-$ любой компакт полосы $D$. Так как для точек $s \in D$ выполняется неравенство $\sigma>1 / 2$, то

$$
\sum_{m=0}^{\infty} \sum_{j=1}^{n} \sup _{s \in K}\left|\frac{a_{m j} b_{m}}{(m+\alpha)^{s}}\right|^{2}<\infty .
$$

Таким образом, остается проверить условие 1) леммы 7.

Пусть $\mu_{1}, \ldots, \mu_{n}-$ комплекснозначные меры на $(\mathbb{C}, \mathscr{B}(\mathbb{C}))$ с компактными носителями, содержащимися в $D$, такие, что

$$
\sum_{m=0}^{\infty}\left|\sum_{j=1}^{n} \int_{\mathbb{C}} \frac{a_{m j} b_{m}}{(m+\alpha)^{s}} d \mu_{j}(s)\right|<\infty .
$$

Если $k$ - наименьшее общее кратное чисел $k_{1}, \ldots, k_{n}$, то оно является периодом всех последовательностей $\mathfrak{a}_{1}, \ldots, \mathfrak{a}_{n}$. Следовательно, ввиду периодичности коэффициентов $a_{m j}$ заключаем из (5), что для $r=1, \ldots, k$

$$
\sum_{\substack{m=0 \\ m \equiv r(\bmod k)}}^{\infty}\left|\sum_{j=1}^{n} a_{r j} \int_{\mathbb{C}} \frac{d \mu_{j}(s)}{(m+\alpha)^{s}}\right|<\infty .
$$

Положим

$$
\nu_{r}(s)=\sum_{j=1}^{n} a_{r j} \mu_{j}(s) .
$$


Тогда $\nu_{r}(s)$ является комплекснозначной мерой на $(\mathbb{C}, \mathscr{B}(\mathbb{C}))$ с компактным носителем, содержащимся в $D, r=1, \ldots, k$. Отсюда и из неравенства (6) имеем, что для всех $r=1, \ldots, k$

$$
\sum_{\substack{m=0 \\ m \equiv r(\bmod k)}}^{\infty}\left|\int_{\mathbb{C}} \frac{d \nu_{r}(s)}{(m+\alpha)^{s}}\right|<\infty .
$$

Пусть

$$
A_{r}=\{m \in \mathbb{N}: m \equiv r(\bmod k)\}
$$

и

$$
\rho_{r}(z)=\int_{\mathbb{C}} e^{-s r} d \nu_{r}(s), \quad z \in \mathbb{C}, \quad r=1, \ldots, k .
$$

Тогда множество $A_{r}$ имеет положительную плотность. Нетрудно видеть, что функция $\rho_{r}(z)$ является целой функцией экспоненциального типа, $r=1, \ldots, k$. Поэтому лемма 8 показывает, что или $\rho_{r}(z) \equiv 0$, или же

$$
\limsup _{x \rightarrow \infty} \frac{\log \left|\rho_{r}(x)\right|}{x}>-1, \quad r=1, \ldots, k .
$$

Здесь мы воспользовались определением полосы $D$ и наличием знака минус в определении $\rho_{r}(z), r=1, \ldots, k$. Если имеет место неравенство $(8)$, то в силу леммы 9

$$
\sum_{m \in A_{r}}\left|\rho_{r}(\log m)\right|=\infty, \quad r=1, \ldots, k .
$$

Хорошо известно, что для всех $s \in \mathbb{C}$

$$
e^{s}=1+O\left(|s| e^{|s|}\right) .
$$

Отсюда находим, что при $m \geqslant 2$

$$
\begin{aligned}
(m+\alpha)^{s} & =m^{-s}\left(1+\frac{\alpha}{m}\right)^{s}=m^{-s} \exp \left\{-s \log \left(1+\frac{\alpha}{m}\right)\right\} \\
& =m^{-s} \exp \left\{\frac{O(|s|)}{m}\right\}=m^{-s}\left(1+\frac{O(|s|)}{m} e^{O(|s|)}\right) \\
& =m^{-s}+m^{-1-\sigma} O(|s|) e^{O(|s|)} .
\end{aligned}
$$

Поскольку компактные носители мер $\nu_{1}, \ldots, \nu_{k}$ содержатся в $D$, отсюда и из неравенства (6) получаем, что

$$
\sum_{m \in A_{r}}\left|\rho_{r}(\log m)\right|<\infty, \quad r=1, \ldots, k,
$$

что противоречит (9). Следовательно,

$$
\rho_{r}(z) \equiv 0, \quad r=1, \ldots, k .
$$

Отсюда и из определений $\rho_{r}(z)$ и $\nu_{r}$ получаем систему уравнений

$$
\sum_{j=1}^{n} a_{r j} \int_{\mathbb{C}} e^{-s z} d \mu_{j}(s) \equiv 0, \quad r=1, \ldots, k .
$$


Поскольку $\operatorname{rank}(A)=n$, то эта система имеет только нулевое решение

$$
\int_{\mathbb{C}} e^{-s z} d \mu_{j}(s) \equiv 0, \quad j=1, \ldots, n .
$$

Отсюда, дифференцируя $r$ раз и полагая $z=0$, получаем, что

$$
\int_{\mathbb{C}} s^{r} d \mu_{j}(s)=0
$$

для всех $j=1, \ldots, n$ и $r \in \mathbb{N}_{0}$. Таким образом, условие 1$)$ леммы 7 также выполняется для последовательности (4). Следовательно, из леммы 7 заключаем, что множество всех сходящихся рядов $(3)$ с $a(m) \in \gamma$ всюду плотно в $H^{n}(D)$. Лемма 6 доказана.

\section{§ 5. Носитель меры $P_{\zeta}$}

Напомним, что носителем меры $P_{\zeta}$ является минимальное замкнутое множество $S_{P_{\zeta}}$ из $H^{n}(D)$ такое, что $P_{\zeta}\left(S_{P_{\zeta}}\right)=1$. Множество $S_{P_{\zeta}}$ состоит из всех таких точек $\underline{g} \in H^{n}(D)$, что для всякой окрестности $G$ элемента $\underline{g}$ выполняется неравенство $P_{\zeta}(G)>0$.

Носителем $S_{X}$ случайного элемента $X$ называется носитель распределения элемента $X$.

Для нахождения носителя меры $P_{\zeta}$ нам понадобится следующее утверждение.

Лемма 10. Пусть $\left\{X_{m}: m \in \mathbb{N}_{0}\right\}$ - последовательность независимых $H^{n}(D)$-значных случайнъх элементов таких, что ряд

$$
\sum_{m=0}^{\infty} X_{m}
$$

сходится почти наверное. Тогда носителем суммы этого ряда является замыкание множества всех таких $g \in H^{n}(D)$, которые могут быть записаны в виде сходящегося ряда:

$$
\underline{g}=\sum_{m=0}^{\infty} \underline{g}_{m}, \quad \underline{g}_{m} \in S_{X_{m}} .
$$

Доказательство см. в [17; лемма 5].

ЛЕмма 11. Носителем случайного элемента $\zeta\left(s, \alpha, \omega ; \mathfrak{a}_{1}, \ldots, \mathfrak{a}_{n}\right)$ является все пространство $H^{n}(D)$.

ДокАЗАТЕЛЬство. Из определения тора $\Omega$ вытекает, что $\left\{\omega(m): m \in \mathbb{N}_{0}\right\}-$ последовательность независимых комплекснозначных случайных величин, определенных на вероятностном пространстве $\left(\Omega, \mathscr{B}(\Omega), m_{H}\right)$. Носителем каждой случайной величины $\omega(m)$ является единичная окружность $\gamma$. Отсюда имеем, что

$$
\left\{\left(\frac{a_{m 1} \omega(m)}{(m+\alpha)^{s}}, \ldots, \frac{a_{m n} \omega(m)}{(m+\alpha)^{s}}\right): m \in \mathbb{N}_{0}\right\}
$$


- последовательность независимых $H^{n}(D)$-значных случайных элементов на $\left(\Omega, \mathscr{B}(\Omega), m_{H}\right)$ и носителем каждого элемента

$$
\left(\frac{a_{m 1} \omega(m)}{(m+\alpha)^{s}}, \ldots, \frac{a_{m n} \omega(m)}{(m+\alpha)^{s}}\right)
$$

является множество

$$
\left\{\underline{g} \in H^{n}(D): \underline{g}(s)=\left(\frac{a_{m 1} a}{(m+\alpha)^{s}}, \ldots, \frac{a_{m n} a}{(m+\alpha)^{s}}\right), a \in \gamma\right\}, \quad m \in \mathbb{N}_{0} .
$$

Следовательно, в силу леммы 10 носителем элемента $\zeta\left(s, \alpha, \omega ; \mathfrak{a}_{1}, \ldots, \mathfrak{a}_{n}\right)$ является замыкание множества всех сходящихся рядов (2). Отсюда и из леммы 6 получаем утверждение леммы 11.

Из леммы 11 и определения носителя случайного элемента получаем, что носителем меры $P_{\zeta}$ также является все пространство $H^{n}(D)$.

\section{§ 6. Доказательство теоремы 1}

Доказательство теоремы 1 основано на леммах 5 и 11, а также на следующем утверждении.

ЛЕмма 12. Пусть $K$ - компактное подмножество комплексной плоскости, обладающее связным дополнением, а функиия $f(s)$ непрерывна на $K u$ аналитична внутри $K$. Тогда $f(s)$ равномерно на $K$ приближается полиномами от $s$.

Лемма 12 является теоремой Мергеляна, ее доказательство см., например, в [28].

ДоКАЗАТЕЛЬСТво ТЕОРЕМЫ 1. МЫ применяем следующие рассуждения. Сначала предположим, что функции $f_{1}(s), \ldots, f_{n}(s)$ аналитически продолжаются на всю полосу $D$. Пусть

$$
G=\left\{\left(g_{1}, \ldots, g_{n}\right) \in H^{n}(D): \sup _{1 \leqslant j \leqslant n} \sup _{s \in K_{j}}\left|g_{j}(s)-f_{j}(s)\right|<\varepsilon\right\} .
$$

Тогда множество $G$ открыто, поэтому из лемм 5 и 11 и свойств слабой сходимости вероятностных мер (см. [21; теорема 2.1]) получаем, что

$$
\liminf _{T \rightarrow \infty} \nu_{T}\left(\sup _{1 \leqslant j \leqslant n} \sup _{s \in K}\left|\zeta\left(s+i \tau, \alpha ; \mathfrak{a}_{j}\right)-f_{j}(s)\right|<\varepsilon\right) \geqslant P_{\zeta}(G)>0 .
$$

Теперь пусть функции $f_{1}(s), \ldots, f_{n}(s)$ удовлетворяют условиям теоремы 1. Тогда в силу леммы 12 существуют полиномы $p_{1}(s), \ldots, p_{n}(s)$ такие, что

$$
\sup _{1 \leqslant j \leqslant n} \sup _{s \in K_{j}}\left|f_{j}(s)-p_{j}(s)\right|<\frac{\varepsilon}{4} .
$$

Так как полиномы - целые функции, то из (10) имеем, что

$$
\liminf _{T \rightarrow \infty} \nu_{T}\left(\sup _{1 \leqslant j \leqslant n} \sup _{s \in K}\left|\zeta\left(s+i \tau, \alpha ; \mathfrak{a}_{j}\right)-p_{j}(s)\right|<\frac{\varepsilon}{2}\right)>0 .
$$


Однако ввиду (11)

$$
\begin{aligned}
\{\tau \in \mathbb{R}: & \left.\sup _{1 \leqslant j \leqslant n} \sup _{s \in K_{j}}\left|\zeta\left(s+i \tau, \alpha ; \mathfrak{a}_{j}\right)-p_{j}(s)\right|<\frac{\varepsilon}{2}\right\} \\
& \subseteq\left\{\tau \in \mathbb{R}: \sup _{1 \leqslant j \leqslant n} \sup _{s \in K_{j}}\left|\zeta\left(s+i \tau, \alpha ; \mathfrak{a}_{j}\right)-f_{j}(s)\right|<\varepsilon\right\}
\end{aligned}
$$

что вместе с (12) влечет за собой утверждение теоремы 1.

Приведем пример периодических дзета-функций Гурвица, удовлетворяющих условиям теоремы 1 . Пусть $a_{01}=2, a_{11}=3, a_{02}=4, a_{12}=5, a_{22}=3$ и последовательность $\mathfrak{a}_{1}=\left\{a_{m 1}: m \in \mathbb{N}_{0}\right\}$ периодична с периодом 2 , а последовательность $\mathfrak{a}_{2}=\left\{a_{m 2}: m \in \mathbb{N}\right\}-$ с периодом 3 . В этом случае $k=6$,

$$
A=\left(\begin{array}{ll}
3 & 5 \\
2 & 3 \\
3 & 4 \\
2 & 5 \\
3 & 3 \\
2 & 4
\end{array}\right) .
$$

Ясно, что $\operatorname{rank}(A)=2$. Пусть $\alpha=e^{-1}$. Тогда функции $\zeta\left(s, \alpha ; \mathfrak{a}_{1}\right)$ и $\zeta\left(s, \alpha ; \mathfrak{a}_{2}\right)$ удовлетворяют условиям теоремы 1.

\section{Список литературы}

[1] С. М. Воронин, “Теорема об "универсальности” дзета-функции Римана”, Изв. АН СССР. Сер. матем., 39:3 (1975), 475-486; англ. пер.: S. M. Voronin, "Theorem on the "universality" of the Riemann zeta-function", Math. USSR-Izv., 9:3 (1975), 443-453.

[2] С. М. Воронин, "Теорема о распределении дзета-функции Римана”, Докл. АН CCCP, 221:4 (1975), 771; англ. пер.: S. M. Voronin, "A theorem on the distribution of values of the Riemann zeta-function", Soviet Math. Dokl., 16:2 (1975), 410.

[3] С. М. Воронин, Аналитические свойства производящих функиий Дирихле арифметических объектов, Дис. ... докт. физ.-матем. наук, МИАН, М., 1977.

[4] С. М. Воронин, "Аналитические свойства производящих функций Дирихле арифметических объектов”, Матем. заметки, 24:6 (1978), 897-884; англ. пер.: S. M. Voronin, "Analytic properties of Dirichlet generating functions of arithmetic objects", Math. Notes, 24:5-6 (1979), 966-969.

[5] С. М. Воронин, "О функциональной независимости $L$-функций Дирихле”, Acta Arith., 27:2 (1975), 493-503.

[6] С. М. Воронин, А.А. Карацуба, Дзета-функиия Римана, Физматлит, М., 1994; англ. пер.: А. А. Karatsuba, S. M. Voronin, The Riemann zeta-function, de Gruyter, Berlin, 1992.

[7] A. Laurinčikas, Limit theorems for the Riemann zeta-function, Kluwer Acad. Publ., Dordrecht, 1996.

[8] K.-G. Grosse-Erdmann, "Universal families and hypercyclic operators", Bull. Amer. Math. Soc., 36 (1999), 345-381.

[9] A. Laurinčikas, "The universality of zeta-functions", Acta Appl. Math., 78:1-3 (2003), 251-271. 
[10] K. Matsumoto, "Probabilistic value-distribution theory of zeta functions", Sugaku Expositions, 17:1 (2004), 51-71.

[11] S. M. Gonek, Analytic properties of zeta and L-functions, Thesis, Univ. Michigan, 1979.

[12] B. Bagchi, The statistical behaviour and universality properties of the Riemann zetafunction and other allied Dirichlet series, Thesis, Indian Statist. Inst., Calcutta, 1981.

[13] B. Bagchi, "A joint universality theorem for Dirichlet $L$-functions", Math. Z., 181 (1982), 319-334.

[14] A. Laurinčikas, K. Matsumoto, "The joint universality and the functional independence for Lerch zeta-functions", Nagoya Math. J., 157 (2000), 211-227.

[15] K. Matsumoto, "Value-distribution of zeta-functions", Analytic number theory (Tokyo, 1988), Lecture Notes in Math., 1434, 1990, 178-187.

[16] А. Лауринчикас, "О нулях линейных комбинаций Матсумото дзета-функций", Литовский матем. сб., 38 (1998), 185-204; англ. пер.: A. Laurinchikas, "On zeros of linear combinations of the Matsumoto zeta functions", Lithuanian Math. J., 38 (1998), 144-159.

[17] A. Laurinčikas, K. Matsumoto, "The joint universality of zeta-functions attached to certain cusp forms", Fiz. Mat. Fak. Moksl. Semin. Darb., 5 (2002), 58-75.

[18] R. Šleževičienè, "The joint universality for twists of Dirichlet series with multiplicative coefficients by characters", Analytic and probabilistic methods in number theory (Palanga, 2001), TEV, Vilnius, 2002, 309-319.

[19] A. Laurinčikas, K. Matsumoto, "The joint universality of twisted automorphic $L$ functions", J. Math. Soc. Japan, 56:3 (2004), 923-939.

[20] A. Laurinčikas, "The joint universality for general Dirichlet series", Ann. Univ. Sci. Budapest. Sect. Comput., 22 (2003), 235-251.

[21] А. Лауринчикас, "Совместная универсальность общих рядов Дирихле", Изв. PАН. Сер. матем., 69:1 (2005), 133-144; англ. пер.: A. Laurincikas, "Joint universality of general Dirichlet series", Izv. Math., 69:1 (2005), 131-142.

[22] A. Javtokas, A. Laurinčikas, On the periodic Hurwitz zeta-function, HardyRamanujan J. (to appear).

[23] A. Javtokas, A. Laurinčikas, "The universality of the periodic Hurwitz zeta-function", Integral Transforms Spec. Funct., 17:10 (2006), 711-722.

[24] К. Прахар, Распределение простых чисел, Мир, М., 1967.

[25] A. Laurinčikas, R. Garunkštis, The Lerch zeta-function, Kluwer Acad. Publ., Dordrecht, 2002.

[26] J. Genys, A. Laurinčikas, "On joint limit theorem of general Dirichlet series", Nonlinear Anal. Model. Control, 8:2 (2003), 27-39.

[27] P. Billingsley, Convergence of probability measures, Wiley, New York, 1968.

[28] J.L. Walsh, Interpolation and approximation by rational functions in the complex domain, Amer. Math. Soc. Colloq. Publ., 20, 1960.

А. Лауринчикас (A. Laurinčikas)

Вильнюсский университет, Литва

E-mail: antanas.laurincikas@maf.vu.lt
Поступила в редакцию 25.01.2006 